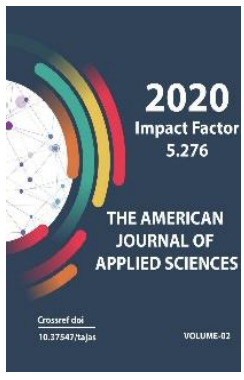

Journal Website: http://usajournalshub.c om/index,php/tajas

Copyright: Original content from this work may be used under the terms of the creative commons attributes 4.0 licence.

\section{Current State And Ways To Improve The Efficiency Of Field Development In The South-Eastern Part Of The Bukhara- Khiva Region}

\author{
Khayitov Odiljon G'afurovich \\ Candidate Of Geological And Mineralogical Sciences, Associate Professor, Head Of The \\ Department "Mining", 100095, Republic Of Uzbekistan, Tashkent, University Street, 2, \\ Tashkent State Technical University, Uzbekistan
}

\title{
ABSTRACT
}

The increase in hydrocarbon production in the Republic as a whole and in the South-Eastern part of the Bukhara-Khiva region is largely determined by the degree of discovery of new fields and the increment of hydrocarbon reserves. The main volume of production and growth of hydrocarbon reserves here is associated with the Jurassic carbonate formation, which for more than 40 years has been the main target object of prospecting, exploration and development of deposits. Due to the relatively high degree of study of the carbonate formation, the probability of discovery of large and medium-sized hydrocarbon deposits in the South-Eastern REGION is low. At the same time, hydrocarbon is increasing the share of small deposits being discovered. To improve the efficiency of development of these deposits and the degree of recovery of hydrocarbon reserves, it is necessary to justify new modern technologies. Since fields with oil deposits have specific features, we will consider this problem separately for oil and gas and gas condensate fields.

\section{KEYWORDS}

Hydrocarbon, oil, gas, deposit, deposits, development efficiency, stage. 


\section{INTRODUCTION}

Analysis of the current state of development of hydrocarbon deposits. Currently, there are several stages in the development of hydrocarbon deposits. At the same time, there are three stages for fields with gas deposits, and 4 stages for oil deposits. Each selected stage of development of hydrocarbon deposits is characterized by certain patterns of changes in technical and economic indicators.

The first stage is the field development stage with drilling of the main well stock and implementation of the reservoir pressure maintenance system. It is characterized by an annual increase in oil production, with a slight water cut of the output of wells. The stage ends when the maximum annual oil or gas production is reached.The secon $d$ is the stage of high extraction rates, varying within $\pm 5 \%$ of the maximum annual oil and gas production. At the end of this stage, there is usually an increase in the water cut of the produced products and the transfer of the well to a mechanized method of operation.

The third stage is a decrease in oil and gas production and a rapid increase in the water content of produced wells. At this stage, annual oil production is $2 \%$ or more of the initial recoverable reserves. The fourth stage is the stage of low rates of oil and gas extraction, high water content of wells produced. At this stage, the rate of oil extraction is usually less than $2 \%$ per year. In practice, the term "late stage" is also widely used, which includes the third and fourth stages of development. As can be seen from table 1 , the hydrocarbon fields discovered on
01.01.2020 are at the spill stages of development.

\section{MATERIAL AND METHODS}

This makes it possible to evaluate the effectiveness of implemented development systems at long-term fields and develop recommendations for improving the efficiency of extracting hydrocarbons from objects at the initial stages of development. From table. 2. it can be seen that there are hydrocarbon deposits in the South-Eastern REGION with both normal hydrostatic pressures and avpd. The current rates of extraction from the initial geological ones are: gas $-1.00 \%$; condensate $0.35 \%$ and oil-0.21\%. 
The American Journal of Applied Sciences

(ISSN - 2689-0992)

Published: September 30, 2020 | Pages: 194-206

Doi: https://doi.org/10.37547/tajas/Volume02Issue09-30

Table 1 Initial geological and recoverable hydrocarbon reserves of the YWCHBHR fields

\begin{tabular}{|c|c|c|c|c|c|c|c|c|}
\hline № & \multirow{2}{*}{ Field } & \multirow{2}{*}{$\begin{array}{c}\text { Productive } \\
\text { horizon }\end{array}$} & \multicolumn{2}{|c|}{ Date } & \multicolumn{3}{|c|}{ Initial geological reserves } & \multirow{2}{*}{$\begin{array}{c}\text { Stage of } \\
\text { developme } \\
n t\end{array}$} \\
\hline $\begin{array}{l}\text { № } \\
\text { P. } \\
\text { p. }\end{array}$ & & & $\begin{array}{l}\text { openin } \\
\text { gs }\end{array}$ & $\begin{array}{l}\text { Introduct } \\
\text { ion to } \\
\text { develop } \\
\text { ment }\end{array}$ & Gaza & $\begin{array}{c}\text { Condens } \\
\text { ate's }\end{array}$ & Oils & \\
\hline 1 & 2 & 3 & 4 & 5 & 6 & 7 & 8 & 9 \\
\hline 1 & Khanabad-N & XV-HP & 2002 & 2018 & - & - & 40 & $\begin{array}{c}\text { In the } \\
\text { concentratio } \\
\text { n camp }\end{array}$ \\
\hline 2 & Sulliger-N & XV-HP & & 2016 & - & - & 55 & The first \\
\hline 3 & Germiston $-\mathrm{N}$ & XV & 1986 & 1992 & - & - & 1980 & Third \\
\hline 4 & Feruza-N & $\mathrm{XV}+\mathrm{XVa}$ & 2002 & 2005 & - & - & 6897 & First \\
\hline 5 & $\begin{array}{l}\text { Maisonminutes } \\
\text {, NGC }\end{array}$ & XV-HP & 2000 & 2010 & - & - & 2362 & First \\
\hline 6 & Karatepa-NGK & $\mathrm{XV}-\mathrm{P}+\mathrm{HP}$ & 2011 & 2013 & 2012 & 176 & 4809 & Fourth \\
\hline 7 & $\begin{array}{l}\text { Yangi Karatepa- } \\
\text { NGC }\end{array}$ & $\begin{array}{l}\mathrm{XV}+\mathrm{XV}- \\
\mathrm{PR}+\mathrm{XVa}\end{array}$ & 2003 & 2005 & 13182 & 2518 & 29118 & Second \\
\hline 8 & $\begin{array}{l}\text { Shakarbulak } \\
\text { NGK }\end{array}$ & $\begin{array}{c}\mathrm{XVa}, \mathrm{XV}- \\
\mathrm{HP}+\mathrm{P}\end{array}$ & 1987 & 1991 & 9071 & 1129 & 32940 & Fourth \\
\hline 9 & Turtsari-NGK & $\begin{array}{c}\mathrm{XV}- \\
\mathrm{HP}+\mathrm{P}+\mathrm{PR}\end{array}$ & 2009 & 2010 & 5142 & 533 & 1545 & Second \\
\hline 10 & $\begin{array}{l}\text { Kumchuk- } \\
\text { NGK }\end{array}$ & $\mathrm{XV}+\mathrm{XVa}$ & 2000 & 2009 & 151 & - & 5902 & $\begin{array}{c}\text { In the } \\
\text { concentratio } \\
n \text { of }\end{array}$ \\
\hline 11 & $\begin{array}{l}\text { SEV. Shurtan } \\
\text { NGK }\end{array}$ & XV-HP+P & 1987 & 2005 & 3357 & 319 & 1268 & Fourth \\
\hline 12 & Ilim-NGK & $\mathrm{XV}+\mathrm{XV}$ & 2007 & 2007 & 3067 & 224 & - & Fourth \\
\hline 13 & $\begin{array}{l}\text { Darakhtli- } \\
\text { NGK }\end{array}$ & XV & 2011 & 2018 & 1792 & - & 133 & $\begin{array}{c}\text { In } \\
\text { developmen } \\
\mathrm{t}\end{array}$ \\
\hline 14 & Kamashi-NGC & $\mathrm{XV}+\mathrm{XVa}$ & 1970 & 2008 & 2408 & 170 & 785 & Third \\
\hline 15 & $\begin{array}{l}\text { Beshkent- } \\
\text { NGK }\end{array}$ & $\mathrm{XV}+\mathrm{XVa}$ & 1974 & 2007 & 11588 & 2422 & 876 & Third \\
\hline 16 & Sherkent-NGK & $\begin{array}{l}X V+X V- \\
P R+X V a\end{array}$ & 2011 & 2011 & 345 & 90 & 2881 & Third \\
\hline 17 & Rubaii-NGK & $\begin{array}{l}\mathrm{XV}+\mathrm{XV}- \\
\mathrm{PR}+\mathrm{XVa}\end{array}$ & 2011 & 2011 & 2134 & 436 & 0 & The first \\
\hline 18 & Aknazar-NGK & $\mathrm{XV}-\mathrm{XVa}$ & 2004 & 2012 & 6382 & 479 & - & The First \\
\hline 19 & $\begin{array}{l}\text { PWM. Aknazar- } \\
\text { NGK }\end{array}$ & XV & 2002 & 2012 & 1085 & - & 4010 & First \\
\hline
\end{tabular}


Notes-the table was compiled by the applicant for geological and field materials of the Shurtan and Mubarek oil and gas production departments listed in the annual geological reports for 2017-2018.

continuation of table1

\begin{tabular}{|c|c|c|c|c|c|c|c|c|}
\hline 1 & 2 & 3 & 4 & 5 & 6 & 7 & 8 & 9 \\
\hline 20 & $\begin{array}{l}\text { Mirmiran- } \\
\text { NGK }\end{array}$ & $\mathrm{XV}-\mathrm{HP}+\mathrm{P}$ & 2007 & 2017 & 639 & - & 422 & $\begin{array}{c}\text { In } \\
\text { conservatio } \\
\text { n }\end{array}$ \\
\hline 21 & $\begin{array}{l}\text { Namesby- } \\
\text { GK }\end{array}$ & $\mathrm{XV}-\mathrm{XVa}$ & 2011 & 2014 & 4346 & of 227.2 & - & Second \\
\hline 22 & Oydin-GC & $\mathrm{XV}-\mathrm{XVa}$ & 2005 & 2011 & 10209 & 393 & - & The first \\
\hline 23 & Chungar-GK & XV-XVa & & 2007 & 13647 & 1138 & - & Second \\
\hline 24 & Shurtan-GC & $\begin{array}{c}\text { XV-PP } \\
+\mathrm{XV}-\mathrm{HP}+\mathrm{P} \\
\end{array}$ & 1974 & 1980 & $\begin{array}{c}64138 \\
3 \\
\end{array}$ & 37200 & - & Fourth \\
\hline 25 & $\begin{array}{l}\text { Ahirbulak- } \\
\text { GC }\end{array}$ & $\begin{array}{c}X V- \\
H P+X V-P\end{array}$ & & & 9471 & & & \\
\hline 26 & $\begin{array}{l}\text { Buzakhur- } \\
\text { GC }\end{array}$ & $\begin{array}{c}\text { XV- } \\
\text { XVa+XVI }\end{array}$ & 1987 & 2005 & 6845 & 419 & - & Third \\
\hline 27 & $\begin{array}{l}\text { East } \\
\text { Buzakhur- } \\
\text { GC }\end{array}$ & $\begin{array}{c}\text { XV- } \\
\text { XVa+XVI }\end{array}$ & 1987 & 2006 & 4113 & 838 & - & Third \\
\hline 28 & $\begin{array}{l}\text { Tarnasky- } \\
\text { GK }\end{array}$ & XV-XVa & ????? & 2012 & 4817 & 299 & - & First page \\
\hline 29 & Tavakkal-GC & $\mathrm{XV}-\mathrm{P}+\mathrm{HP}$ & 2010 & 2010 & 6200 & 235 & - & $\begin{array}{c}\text { In the } \\
\text { exploration }\end{array}$ \\
\hline 30 & $\begin{array}{l}\text { Alachagikud } \\
\text { uk-GC }\end{array}$ & XV-P+ HP & & & 375 & & & $\begin{array}{c}\text { In the } \\
\text { exploration }\end{array}$ \\
\hline 31 & Zafar-GC & $\mathrm{XV}+\mathrm{XVa}$ & ??? & 1994 & 3059 & 877 & - & Second \\
\hline 32 & $\begin{array}{l}\text { SEV.NIshan- } \\
\text { GC }\end{array}$ & $\begin{array}{c}\mathrm{XV}+\mathrm{XVa}+ \\
\mathrm{XVI}\end{array}$ & 1970 & 2007 & 30366 & 1761 & - & Third \\
\hline 33 & Nishan-GC & $\mathrm{XV}-\mathrm{XVa}$ & 2008 & 2011 & 2098 & ???? & - & Third \\
\hline 34 & $\begin{array}{l}\text { Sept.Guzar } \\
\text { GC }\end{array}$ & $\mathrm{XV}-\mathrm{HP}+\mathrm{P}$ & 1992 & 2007 & 8471 & 1735 & - & Third \\
\hline 35 & $\begin{array}{l}\text { Hotel } \\
\text { marvarid - } \\
\text { GK }\end{array}$ & $\mathrm{XV}$ & & & 894 & & & \\
\hline 36 & $\begin{array}{l}\text { Topicxal - } \\
\text { GK }\end{array}$ & & & & 1072 & & & \\
\hline 37 & Giran GK & $\begin{array}{c}\mathrm{XV}+\mathrm{XVa}+ \\
\mathrm{XVI}\end{array}$ & 1979 & 1989 & 10604 & 625 & - & The Third \\
\hline
\end{tabular}


The American Journal of Applied Sciences

(ISSN - 2689-0992)

Published: September 30, 2020 | Pages: 194-206

Doi: https://doi.org/10.37547/tajas/Volume02Issue09-30

\begin{tabular}{|c|l|c|c|c|c|c|c|c|}
\hline 38 & $\begin{array}{l}\text { PWM.Girsan } \\
\text { GC }\end{array}$ & $\begin{array}{c}\text { XV+XVa+ } \\
\text { XVI }\end{array}$ & 2009 & 2010 & 12643 & 715 & - & The first \\
\hline 39 & $\begin{array}{l}\text { of Divana } \\
\text { GK }\end{array}$ & $\begin{array}{l}\text { XV+XVa+ } \\
\text { XVI }\end{array}$ & 2009 & 2010 & 1891 & 109,550 & - & First \\
\hline 40 & Ernazar-GC & XV -XVa & 2009 & 2010 & 8209 & 299 & - & First \\
\hline 41 & Chigil-GC & XV-XVa+ & 2008 & 2010 & 17889 & 898 & - & First \\
\hline 42 & $\begin{array}{l}\text { Talimarjon - } \\
\text { GK }\end{array}$ & XV-XVa+ & 2009 & 2019 & 16854 & 618 & - & The first \\
\hline 43 & $\begin{array}{l}\text { Nazarchuk - } \\
\text { GK }\end{array}$ & XV-XVa+ & 2009 & 2012 & 11127 & 563 & - & First \\
\hline 44 & Kapali-G & XV-XVa & & & 386 & & & \\
\hline
\end{tabular}

Table 2.

State of hydrocarbon production in the South CAUCASUS Federal district

\begin{tabular}{|c|c|c|c|c|c|c|c|c|}
\hline \multirow{2}{*}{$\begin{array}{l}\text { № } \\
\text { № } \\
\text { Ite } \\
\text { m } \\
\text { no }\end{array}$} & \multirow[t]{2}{*}{ Field } & \multicolumn{2}{|c|}{$\begin{array}{c}\text { Reservoir pressure, } \\
\mathbf{k g} / \mathrm{cm}^{2}\end{array}$} & \multicolumn{2}{|c|}{$\begin{array}{c}\text { Fund of drilled } \\
\text { wells }\end{array}$} & \multicolumn{3}{|c|}{ Annual production } \\
\hline & & Initial & $\begin{array}{c}\text { Current } \\
\text { state }\end{array}$ & Total & Current & $\begin{array}{c}\text { Gas } \\
\text { consumpti } \\
\text { on, mln. } \\
\mathbf{m}^{\mathbf{3}}\end{array}$ & $\begin{array}{l}\text { Conde } \\
\text { nsate, } \\
\text { thousa } \\
\text { nd } \\
\text { tons of }\end{array}$ & $\begin{array}{c}\text { Oil } \\
\text { thousand } \\
\text { tons }\end{array}$ \\
\hline 1 & 2 & 3 & 4 & 5 & 6 & 7 & 8 & 9 \\
\hline 1 & Khanabad-N & 350 & 281 & 3 & - & - & - & - \\
\hline 2 & Soligor-N & 310 & 300 & 5 & - & - & - & 2,685 \\
\hline 3 & Hermiston -N & 352 & 240 & 17 & 6 & 6,537 & - & 35,626 \\
\hline 4 & Feruza-N & 486 & 292 & 9 & 4 & - & - & 0,733 \\
\hline 5 & Meson, NGC & 317 & 270 & 4 & - & - & - & 0,008 \\
\hline 6 & $\begin{array}{l}\text { Karatepa- } \\
\text { NGK }\end{array}$ & 503 & 124 & 14 & 11 & 89,226 & - & 17,110 \\
\hline 7 & \begin{tabular}{l|} 
Yangi \\
Karatepa NGK
\end{tabular} & 504,5 & 124 & 17 & 2 & 27,515 & 0,44 & 1,238 \\
\hline 8 & $\begin{array}{l}\text { of } \\
\text { Shakarbulak } \\
\text { NGK }\end{array}$ & 395 & 296,8 & 32 & 9 & 71,099 & 0,751 & 18,872 \\
\hline 9 & $\begin{array}{l}\text { Curtsey- } \\
\text { NGK }\end{array}$ & 355 & 218 & 7 & 5 & 58,160 & - & 10,874 \\
\hline 10 & $\begin{array}{l}\text { Kumchuk- } \\
\text { NGK }\end{array}$ & 381 & 208 & 5 & - & - & - & - \\
\hline 11 & $\begin{array}{l}\text { SEV. Shurtan } \\
\text { NGK }\end{array}$ & 387 & 140 & 25 & 9 & 2,577 & 77,6 & 86,871 \\
\hline 12 & Ilim-NGK & 383,5 & 49,6 & 7 & 5 & 93,013 & 3,6 & 1,012 \\
\hline 13 & Darakhtli- & - & - & - & - & - & - & \\
\hline
\end{tabular}


The American Journal of Applied Sciences

(ISSN - 2689-0992)

Published: September 30, 2020 | Pages: 194-206

Doi: https://doi.org/10.37547/tajas/Volumeo2Issue09-30

\begin{tabular}{|c|c|c|c|c|c|c|c|c|}
\hline & NGK & & & & & & & \\
\hline 14 & $\begin{array}{l}\text { Kamashi- } \\
\text { NGC }\end{array}$ & 571,5 & 318 & 8 & 5 & 11,767 & 0.191 & 3,972 \\
\hline 15 & $\begin{array}{l}\text { Beshkent- } \\
\text { NGK }\end{array}$ & 573,4 & 301 & 12 & 4 & 5,492 & 0.125 & 6,261 \\
\hline 16 & $\begin{array}{l}\text { Sherkent- } \\
\text { NGK }\end{array}$ & 469 & 352 & 6 & 1 & 0.293 & & 0.908 \\
\hline 17 & Ruboi-NGK & 486 & 361 & 8 & 6 & 29,205 & 0,424 & 6,626 \\
\hline 18 & $\begin{array}{l}\text { Aknazar- } \\
\text { NGK }\end{array}$ & 623 & 182 & 6 & 1 & 4,906 & - & 0,153 \\
\hline 19 & $\begin{array}{l}\text { PWM. } \\
\text { Aknazar-NGK }\end{array}$ & 621 & 331 & 4 & 1 & - & - & 0,19 \\
\hline 20 & $\begin{array}{l}\text { Mirmiran- } \\
\text { NGK }\end{array}$ & & & & & & & \\
\hline 21 & $\begin{array}{l}\text { Namazbay- } \\
\text { group }\end{array}$ & $\begin{array}{c}\text { of } \\
\text { companie } \\
\text { s } 321\end{array}$ & 143 & 8 & 6 & 39 & 1,121 & - \\
\hline 22 & Oydin-group & $\begin{array}{c}\text { of } \\
\text { companie } \\
\text { s 271,6 }\end{array}$ & 181,7 & 3 & 1 & 7,315 & 0,20 & - \\
\hline 23 & $\begin{array}{l}\text { Chunagar- } \\
\text { group }\end{array}$ & $\begin{array}{c}\text { of } \\
\text { companie } \\
\text { s } 344\end{array}$ & 91,2 & 17 & 2 & 42,860 & 1,4 & - \\
\hline 24 & $\begin{array}{l}\text { Shurtan- } \\
\text { group }\end{array}$ & $\begin{array}{c}\text { of } \\
\text { companie } \\
\text { s } 349 \\
\end{array}$ & 44,2 & 239 & 151 & 6634,416 & 189 & - \\
\hline 25 & $\begin{array}{l}\text { Ahirbulak- } \\
\text { GC }\end{array}$ & & & & & & & \\
\hline 26 & $\begin{array}{l}\text { Buzakhur- } \\
\text { GC }\end{array}$ & 365 & 45,02 & 19 & 7 & 123,660 & - & - \\
\hline 27 & $\begin{array}{l}\text { East } \\
\text { Buzakhur- } \\
\text { group of } \\
\text { companies }\end{array}$ & $\begin{array}{c}\text { Buzakhur } \\
\text {-group of } \\
\text { companie } \\
\text { s } 346\end{array}$ & 76 & 12 & 2 & 28 & 1,3 & - \\
\hline 28 & $\begin{array}{l}\text { Tarnasoy- } \\
\text { group }\end{array}$ & $\begin{array}{c}\text { of } \\
\text { companie } \\
\text { s } 368 \\
\end{array}$ & 87 & 2 & 1 & 28 & 0,398 & - \\
\hline 29 & Tavakkal- & $\begin{array}{c}\text { group of } \\
\text { companie } \\
\text { s } 306,8\end{array}$ & 310 & 6 & - & - & - & - \\
\hline 30 & $\begin{array}{l}\text { Alachagikud } \\
\text { uk-GC }\end{array}$ & & & & & & & \\
\hline 31 & Zafar-GC & 431 & 50,9 & 12 & 2 & 25,200 & 0,7 & - \\
\hline 32 & SEV.NIshan- & $\begin{array}{l}\text { group of } \\
\text { companie }\end{array}$ & 118 & 50 & 32 & 323,264 & 7,095 & - \\
\hline
\end{tabular}


The American Journal of Applied Sciences

(ISSN - 2689-0992)

Published: September 30, 2020 | Pages: 194-206

Doi: https://doi.org/10.37547/tajas/Volume02Issue09-30

\begin{tabular}{|c|c|c|c|c|c|c|c|c|}
\hline & & s 550 & & & & & & \\
\hline 33 & Nishan-GC & & & & & 8,099 & - & \\
\hline 34 & $\begin{array}{l}\text { SEV.Guzar } \\
\text { group }\end{array}$ & $\begin{array}{c}\text { of } \\
\text { companie } \\
\text { s } 317\end{array}$ & 105 & 13 & 5 & 38,384 & 0,544 & - \\
\hline 35 & Marvarid-GC & & & & & & & - \\
\hline 36 & $\begin{array}{l}\text { Topicxal - } \\
\text { GK }\end{array}$ & & & & & & & \\
\hline 37 & GK Giran & 621 & 190 & 12 & 8 & 281,9 & 15,2 & - \\
\hline 38 & $\begin{array}{l}\text { PWM.Girsan } \\
\text { group }\end{array}$ & $\begin{array}{c}\text { of } \\
\text { companie } \\
\text { s } 598\end{array}$ & 306 & 7 & 4 & 39,2 & 0,179 & - \\
\hline 39 & $\begin{array}{l}\text { Divkhana } \\
\text { group }\end{array}$ & $\begin{array}{c}\text { of } \\
\text { companie } \\
\text { s } 548\end{array}$ & 210,6 & 6 & 4 & 27,2 & 0,788 & - \\
\hline 40 & Ernazar- & $\begin{array}{c}\text { group of } \\
\text { companie } \\
\text { s } 629 \\
\end{array}$ & 304,8 & 16 & 13 & 513,7 & 17,47 & - \\
\hline 41 & Chigil-GC & 615 & 308 & 7 & 4 & 24,5 & 0,735 & \\
\hline 42 & $\begin{array}{l}\text { Talimarjon- } \\
\text { GC }\end{array}$ & 457 & 352 & 13 & 9 & 203,6 & 6,116 & \\
\hline 43 & $\begin{array}{l}\text { Nazarchuk - } \\
\text { GK }\end{array}$ & 630 & 256,8 & 4 & 2 & 153,4 & 5,432 & \\
\hline 44 & Kapali-G & & & & & & & \\
\hline
\end{tabular}

\section{RESULTS}

Potential amounts of gas, condensate and oil extraction indicate a low intensity of development systems implemented at the fields.

The magnitude of the rate of gas and condensate without regard to the unique field of Shurtan is 0.93 , and 0.70 percent respectively.

As you know, the generally accepted indicator for evaluating the efficiency of hydrocarbon field development is the recovery coefficients of gas, condensate and oil from productive formations.
The values оfуглеводородов these indicators vary within very large limits depending on the stage of their development at the Yakh hydrocarbon fields of the South Siberian oil and gas industry (table 3), averaging: gas recovery factor (GRI) - 0.626; condensate recovery factor $(\mathrm{CIC})-0.429$; oil recovery factor (OC) - 0.037. Current (as of 01.01.2020), $\mathrm{KIC}$ and $\mathrm{KIC}$ values excluding the Shurtan field are 0.224 and 0.168 , respectively. The obtained values of KIG, KIC and KIN indicate that the development systems implemented in the YVCHBKHR hydrocarbon fields were generally ineffective.

In this regard, we will consider the reasons for the low efficiency of field development and 
The American Journal of Applied Sciences

(ISSN - 2689-0992)

Published: September 30, 2020 | Pages: 194-206

Doi: https://doi.org/10.37547/tajas/Volume02Issue09-30

possible ways to improve them. Analysis of the current state and ways to increase the efficiency of oil and gas fields development in the South-Eastern part of the Bukhara-Khiva region.
Oil and gas fields account for the majority of oil reserves on the territory of the South Caucasus Federal DISTRICT. In which oil deposits are located under a gas cap relatively with a more powerful effective thickness.

Table 3

The state proficiencies of depletion of hydrocarbonreserves in the South West of the RUSSIAN Federation

\begin{tabular}{|l|l|c|c|c|c|c|c|}
\hline \multirow{2}{*}{$\begin{array}{c}\text { № } \\
\text { Ino } \\
\text { Ite } \\
\text { mo. }\end{array}$} & \multicolumn{1}{|c|}{ Field } & \multicolumn{3}{|c|}{ Accumulated production } & \multicolumn{2}{c|}{$\begin{array}{c}\text { The current recovery factor, } \\
\text { fractions of one. }\end{array}$} \\
\cline { 2 - 8 } & $\begin{array}{c}\text { Gas, } \\
\text { million } \\
\text { m3 }^{3}\end{array}$ & $\begin{array}{c}\text { Condens } \\
\text { ate, } \\
\text { thousand } \\
\text { tons }\end{array}$ & $\begin{array}{c}\text { of Oil, } \\
\text { thousand tons } \\
\text { of gas. }\end{array}$ & Gas & $\begin{array}{c}\text { Condensat } \\
\text { e's. }\end{array}$ & Oil \\
\hline 1 & \multicolumn{1}{|c|}{2} & 3 & 4 & 5 & 6 & 7 & 8 \\
\hline 1 & Khanabad-N & - & - & 1,524 & - & - & 0,044 \\
\hline 2 & Sovligar-N & - & - & 3,951 & - & - & 0,072 \\
\hline 3 & Garmiston -N & - & - & 524,615 & - & - & 0,265 \\
\hline 4 & Feruza-N & - & - & 81,142 & - & - & 0,011 \\
\hline 5 & Meson, NGC & - & - & 4,322 & - & - & 0,090 \\
\hline 6 & $\begin{array}{l}\text { Karatepa- } \\
\text { NGK }\end{array}$ & 2713,103 & 340,6275 & 130,711 & 0,534 & 0,331 & 0,080 \\
\hline 7 & $\begin{array}{l}\text { Yangi Karatepa- } \\
\text { NGK }\end{array}$ & 2741 & 341,070 & 130,712 & 0.2122 & 0.1990 & 0.3610 \\
\hline 8 & $\begin{array}{l}\text { Shakarbulok } \\
\text { NGK }\end{array}$ & 1632 & 33 & 697 & 0.180 & 0.029 & 0.021 \\
\hline 9 & Turtsari-NGK & 850 & 25.164 & 117,984 & 0.1790 & 0.0629 & 0.1071 \\
\hline 10 & $\begin{array}{l}\text { Kumchuk- } \\
\text { NGK }\end{array}$ & - & - & 8,671 & - & - & 0,001 \\
\hline 11 & $\begin{array}{l}\text { SEV. Shurtan } \\
\text { NGK }\end{array}$ & 766 & 31,004 & 1210,012 & 0.6978 & 0.6420 & 0.1574 \\
\hline 12 & Ilim-NGK & 2098 & 114,563 & - & 0,6833 & 0,6160 & - \\
\hline 13 & $\begin{array}{l}\text { Darakhtli- } \\
\text { NGK }\end{array}$ & - & - & - & - & - & - \\
\hline 14 & Kamashi-NGC & 641 & 37,154 & 132 & 0.266 & 0.219 & 0.169 \\
\hline 15 & $\begin{array}{l}\text { Beshkent- } \\
\text { NGK }\end{array}$ & 6190 & 466,062 & 181,101 & 0.534 & 0.192 & 0.206 \\
\hline 16 & Sherkent-NGK & 169 & 5.649 & 178.823 & 0.4899 & 0.0926 & 0.2436 \\
\hline 17 & Ruboyi-NGK & 48 & 0,727 & 27,650 & 0,022 & 0,002 & - \\
\hline & & & & & & \\
\hline
\end{tabular}


The American Journal of Applied Sciences (ISSN - 2689-0992)

Published: September 30, 2020 | Pages: 194-206

Doi: https://doi.org/10.37547/tajas/Volume02Issue09-30

\begin{tabular}{|c|l|c|c|c|c|c|c|}
\hline 18 & Aknazar-NGK & 56,593 & - & 1,776 & 0,010 & 0,004 & - \\
\hline 19 & $\begin{array}{l}\text { PWM. Aknazar- } \\
\text { NGK }\end{array}$ & - & - & 3,924 & - & - & 0,003 \\
\hline
\end{tabular}

Continuation of table 3

\begin{tabular}{|c|c|c|c|c|c|c|c|}
\hline 1 & 2 & 3 & 4 & 5 & 6 & 7 & 8 \\
\hline 20 & $\begin{array}{l}\text { Mirmiran- } \\
\text { NGK }\end{array}$ & - & - & - & - & - & - \\
\hline 21 & Namazbay- & $\begin{array}{l}\text { group of } \\
\text { compani } \\
\text { es } 448\end{array}$ & 19718 & - & 0,1050 & 0,1084 & - \\
\hline 22 & Oydin-group & $\begin{array}{c}\text { of } \\
\text { compani } \\
\text { es } 105\end{array}$ & 5,024 & - & 0,0141 & 0,0244 & - \\
\hline 23 & $\begin{array}{l}\text { Chunagar- } \\
\text { group }\end{array}$ & $\begin{array}{c}\text { of } \\
\text { compani } \\
\text { es } 1367\end{array}$ & 60,543 & - & 0,1021 & 0,063 & - \\
\hline 24 & Shurtan-group & $\begin{array}{c}\text { of } \\
\text { compani } \\
\text { es } \\
501061\end{array}$ & $\begin{array}{c}21242,21 \\
2\end{array}$ & - & 0,7901 & 7165 & - \\
\hline 25 & Akhirbulak- & $\begin{array}{c}\text { group of } \\
\text { compani } \\
\text { es - }\end{array}$ & - & - & - & - & - \\
\hline 26 & Buzakhur-GC & 6739 & 331,646 & - & 0,9963 & 0,9476 & - \\
\hline 27 & $\begin{array}{l}\text { East } \\
\text { Buzakhur- }\end{array}$ & $\begin{array}{l}\text { group of } \\
\text { compani } \\
\text { es } 1143\end{array}$ & 164,722 & - & 0,2906 & 0,3050 & - \\
\hline 28 & $\begin{array}{l}\text { Tarnasoy- } \\
\text { group }\end{array}$ & $\begin{array}{c}\text { of } \\
\text { compani } \\
\text { es } 228\end{array}$ & 3,544 & - & 0,0479 & 0,0142 & - \\
\hline 29 & $\begin{array}{l}\text { Tavakkal- } \\
\text { group }\end{array}$ & of & $\begin{array}{c}\text { companie } \\
\text { s } 77,184 \\
2,527 \\
\end{array}$ & - & 0,012 & 0,010 & - \\
\hline 30 & $\begin{array}{l}\text { Alachagikudu } \\
\text { k-group }\end{array}$ & $\begin{array}{c}\text { of } \\
\text { compani } \\
\text { es - }\end{array}$ & - & - & - & - & - \\
\hline 31 & Zafar-group & $\begin{array}{c}\text { of } \\
\text { compani } \\
\text { es } 772\end{array}$ & 111,888 & - & 0,2678 & 0,1955 & - \\
\hline 32 & SEV.NIshan- & $\begin{array}{l}\text { group of } \\
\text { compani } \\
\text { es } 14931\end{array}$ & 619 & - & 0,4917 & 0,3515 & - \\
\hline 33 & Nishan-GC & 8,099 & - & - & & & \\
\hline
\end{tabular}


The American Journal of Applied Sciences

(ISSN - 2689-0992)

Published: September 30, 2020 | Pages: 194-206

Doi: https://doi.org/10.37547/tajas/Volumeo2Issue09-30

\begin{tabular}{|c|c|c|c|c|c|c|c|}
\hline 34 & $\begin{array}{l}\text { SEV.Guzar } \\
\text { group }\end{array}$ & $\begin{array}{c}\text { of } \\
\text { compani } \\
\text { es } 2987\end{array}$ & 382,4 & - & 0,352 & 0,220 & - \\
\hline 35 & $\begin{array}{l}\text { Hotel } \\
\text { marvarid - } \\
\text { GK }\end{array}$ & & & & & & \\
\hline 36 & $\begin{array}{l}\text { Topichaksoy- } \\
\text { GC }\end{array}$ & & & & & & \\
\hline 37 & Girsan GC & 5835,4 & 192,9 & - & 0,551 & 0,310 & - \\
\hline 38 & $\begin{array}{l}\text { PWM.Girsan } \\
\text { group }\end{array}$ & $\begin{array}{c}\text { of } \\
\text { compani } \\
\text { es } 135 \\
\end{array}$ & 7,539 & - & 0,0289 & 0,0134 & - \\
\hline 39 & $\begin{array}{l}\text { Divkhana } \\
\text { group }\end{array}$ & $\begin{array}{c}\text { of } \\
\text { compani } \\
\text { es } 316\end{array}$ & 16,552 & - & 0,1707 & 0,1602 & - \\
\hline 40 & Ernazar-group & $\begin{array}{c}\text { of } \\
\text { compani } \\
\text { es } 1704 \\
\end{array}$ & 73,754 & - & 0,3816 & 0,1715 & - \\
\hline 41 & Chigil-group & $\begin{array}{c}\text { of } \\
\text { compani } \\
\text { es } 31,4\end{array}$ & 0,9 & - & 0,002 & 0,001 & - \\
\hline 42 & $\begin{array}{l}\text { Talimarjon- } \\
\text { group }\end{array}$ & of & $\begin{array}{c}\text { companie } \\
\text { s } 271,182 \\
11,381\end{array}$ & - & 0,016 & 0,018 & - \\
\hline 43 & $\begin{array}{l}\text { Nazarkuduk- } \\
\text { group }\end{array}$ & of & $\begin{array}{c}\text { companie } \\
\text { s } 304,945 \\
11,7897\end{array}$ & - & 0,0303 & 0,072 & - \\
\hline 44 & Kapali-G & & & & & & \\
\hline
\end{tabular}

Due to the high complexity of the wells due to the breakthrough of gas in the oil of these objects wells due to the breakthrough of gas from a gas cap and bottom water to downhole stocks of these objects belong to the category of hard-to-recover [3; p. 46-50, 4; p. 267, 5; p. 34-36, etc.].

Sub-gas oil deposits are usually developed with the prevailing water-pressure, gas $า-$ pressure or simultaneous manifestation of both modes. As is well known, when developing a field using the technology of oil displacement by various agents, the oil recovery factor (EIR) is calculated using the modified formula of A. N. Krylov [6; p. 15-17].
КИН $=$ Квыт $\bullet$ Кохв $=$ Квыт $\bullet$ Кохв. $\sqcap$ Кохв. $\mathrm{T}$

(1)

where Toinyt. - coefficient of displacement of oil by the working agent; KKSHV. p, Kinyt. tкоэффилсоefficients of displacement coverage in the area and thickness of the formation, respectively.

In the development of fields with heterogeneous reservoirs, the value OfCW. $p$ is mainly influenced by the density of the grid of wells and the scheme of their placement. In this regard, in a number of studies, this coefficient is called 7 the grid coefficient [7; p.15-18, 8; p. 34-37], the value of which 
depends on the density of the adopted grid of wells placement ( $\mathrm{S}$ - the area of oil content per well), on the zonal heterogeneity and discontinuity of oil reservoirs.

Due to the great practical importance of this issue, many scientists and research centers have conducted studies to determine the dependence oil recovery on the density of the well grid (PSS). Dependencies were obtained both for specific fields and for oil-producing regions. The disadvantages of these dependencies are the complexity of defining their parameters, $\urcorner$ which is why they are not widely used.

\section{DISCUSSIONS}

It should be noted that there are also such methods for estimating the-coefficient of coverage of wells with a grid - the "kh-ratio method" and " method Stiles, $\neg$ which are used to justify and analyze the potential of compacting drilling. The main disadvantage of these methods is also the complexity in determining 7 parameters, in particular the uncertainty in constructing the connectivity distance relationship between wells, which is caused by a large spread of points obtained using the proposed algorithms [9; p. 40-45]. The determination of a more reasonable value of $\mathrm{KC}$ is not a big problem if there is a hydrodynamic model of the Deposit 7 that correctly reproduces the features of the structure of the Deposit. In this ᄀregard, the development and approval of a guidance document on the creation of a permanent geological and technological model of a hydrocarbon field in the design of the development of oil and gas fields in Uzbekistan is timely [10; 80c.]. ᄀThe use of this guidance document ( $\mathrm{RH}$ 39.0-105:2012) will speed up the implementation of advanced computer technologies in the design 7 and management of oil and gas and oil field development, and allow for the operation of geologic and technological information in its entirety (3D), taking into account changes over time (4D).

However, the uncertainty and lack of input data required for $\neg$ a permanent geological and technological model and for their ᄀinitialization may limit the use of 3Dsimulators for predicting both the oil recovery coefficient and the well grid coefficient, especially at the stage of field development and the calculation of hydrocarbon reserves. In this regard, it is necessary to focus attention on another problemathat is characteristic of the initial stage of field development. As you know, the first document on the new field is the calculation of hydrocarbon reserves.

\section{CONCLUSIONS}

Naturally, in the initial stage, special emphasis is placed on the types of research $\urcorner$ aimed at determining and justifying the estimated parameters $\urcorner$ of carbon dioxide reserves. This leads to the fact that in the preparation of project documents for development of oil fields with application of hydrodynamic calculation methods, based on mathematical description of mechanism of process oil recovery and requires the use of a wide range of settings, acute lacked $\neg$ the ka of data on reservoir permeability, the change in the value of oil properties and gas pressure, phase and relative permeability, of the geological heterogeneity parameters of layers etc. Under these conditions, the use empirical formulas becomes not only unavoidable, but also significant for making management decisions in the initial stage of development. 


\section{REFERENCES}

1. Hayitov O. agzamova S. A., Muhammad H. M.. Determination of coefficient of appointest reservoir of the North field of Urtabulak according for the Deposit exploitation in the early stages. // Innovative development of oil and gas geological science of Uzbekistan and the role of youth in solving its problems ("Akramkhodzhayevskie chteniya"). T.: OA "IGIRNIGM". 2018. - Pp. 101-104.

2. Karshiev O. A., Boydedaev A. A., Zorin A. A., Derevyanko T. V., Mamadaliev B. T. Prospects of oil and gas content of Jurassic terrigenous deposits of the Beshkent trough and the SouthWestern spurs of the Hissar ridge / / Oil and gas Geological science of Uzbekistan and the role of youth in solving its problems// Materials of the scientific conference. "Works of young scientists. Issue 4". - Tashkent: JSC "IGIRNIGM", 2015. - 132 p.

3. Hayitov O. G., Nabiyev N. To., Mahmudov Sh. N. The evaluation of the impact of well spacing on oil recovery factor oil of oil deposits. |/»|zvestiya vuzov. Mining journal». Uralsky: 2013, No. 6, Pp. 46-50.

4. Abdullaev G. S. Features of the geological structure of the Jurassic carbonate deposits of the Northflowing part of the Beshkent trough and associated prospects for oil and gas potential. - T.: Uzbek journal of oil and gas, 2010. no. 4. - P. 10-12.

5. Muratov A. S. Prospects for detecting oil and gas deposits in the Northern part of the Beshkent trough of the Bukhara-Khivn region. - T.: Uzbek journal of oil and gas, 2010, no. 4. - P. 13-16.

6. Evseeva G. B. the Role of foraminifera in the development of stratigraphic schemes of the Jurassic carbonate formation of the Bukhara-Khivn region. - T.: Uzbek journal of oil and gas, 2007, no. 3. - P. 23-26.

7. Karshiev O. A., Abbasova S. A. Features of the zonal change of the "uglevodorod-Voda" contact in the Western part of the Beshkent deflection of the Kamashinsky Val and Karatepinsky uplift. - T.: Uzbek journal of oil and gas, 2014, no. 4. - Pp. 14-17.

8. Karshiev O. A., Abbasova S. A. New data on the geological structure and oil and gas content of the Eastern slope of the Dengizkul subdistrict of the Bukhara-Khivn region. - T.: Uzbek journal of oil and gas, 2016, no. 4. - Pp. 20-24

9. Megliev F. E. Features of the tectonic structure of the North-Eastern part of the Beshkent trough and its connection with oil and gas capacity. T.: Uzbek journal of oil and gas, 2005, no. 3. - Pp. 10-13.

10. Megliev F. E., Gulyamova A. K. Isolation and development of complex reservoirs in carbonate rocks of the Beshkent trough. - T.: Uzbek journal of oil and gas, 2003, no. 2. - P. 13-15.

11. Lysenko V. D. Problems of designing the development of zonal heterogeneous oil fields. 2007. No. 11. Pp. 15-17.

12. Agzamov A. A., khayitov O. G., karshiev $A$. $H$. on the degree of influence of the rate of liquid selection on the rate of oil selection at various stages of development of deposits represented by carbonate reservoirs. // 
"Izvestiya vuzov. Mining journal». Uralsky. 2016. no. 4-P. 36-46.

13. Hayitov O. G. Yusupkhodzhaeva E.N. Abdurakhmanova S.P. Halmatova G.N. ON THE STATE OF HYDROCARBON RESOURCE BASE IN THE BESHKENT TROUGH On the state of hydrocarbon resource base in the Beshkent trough. -P-2327-2331.

https://www.jardcs.org/abstract.php?i $\mathrm{d}=5787$

14. Khayitov O. G. on formation of abnormally high and abnormally low reservoir pressures. - //VI Международная научнопрактическая конференция. «GLOBAL SCIENCE AND INNOVATIONS 2019: CENTRAL ASIA». NUR-SULTAN2019. IX Vol. - P. 82-86.

15. Yudin E. V., Lubnin A. A., roschektaev A. P. Estimation of the grid coverage coefficient using well operation data / / Territory "NEFTEGAZ". 2011. no. 4. - P. 40-45. 